\title{
THE BLOOD FLOW IN THE RIGHT ATRIUM AND SUPERIOR VENA CAVA IN TRICUSPID INCOMPETENCE
}

\author{
BY \\ OTTAR MÜLLER* AND JOHN SHILLINGFORD $\dagger$ \\ From the Department of Medicine, Postgraduate Medical School, Hammersmith, London
}

Received November 11, 1954

In the course of a study of tricuspid incompetence (Müller and Shillingford, 1954) it was found that a high mean venous pressure in congestive cardiac failure was frequently associated with an abnormal right atrial pressure curve. Analysis of a series of right atrial pressure curves in normal subjects and patients with cardiac failure (Korner and Shillingford, 1954) showed in the latter group a progressive change in their form as the mean right atrial pressure rose; with moderate rises of venous pressure there was impairment of the $x$ descent, and in the presence of a greater mean pressure, a positive systolic wave was found. In view of these findings we have made further studies on the blood flow in the right atrium and superior vena cava to elucidate the part played by functional tricuspid incompetence in the altered hæmodynamics of cardiac failure.

The purpose of this paper is to describe a technique for the measurement of blood flow in the atrium and superior vena cava in man, and to show the changes that occur with the development of tricuspid incompetence.

\section{METHODS}

Thirteen patients were studied; in five there was no evidence of tricuspid incompetence and in eight a tricuspid leak was diagnosed clinically and confirmed by right atrial pressure records.

A Cournand double lumen catheter, with one hole at the tip and the other at the side $10 \mathrm{~cm}$. back from the first, was directed via the ante-cubital vein into the right atrium such that the end hole was two or three $\mathrm{cm}$. inside the right atrium and the side hole in the superior vena cava. The catheter was connected to a sensitive differential manometer (Müller and Shillingford, 1954), the output of which was amplified by a direct current amplifier and recorded on an Elmqvist electrocardiograph. The recording system was so arranged that if the pressure were higher in the right atrium than the superior vena cava the deflection would be below the zero line, if the pressures were equal there would be no deflection, and if the pressure in the superior vena cava were higher than in the right atrium the deflection would be above the line.

The interpretation of the curves so recorded was based on experiments carried out in a heart circulation model (Fig. 1); this consisted of a rubber balloon suspended in a glass chamber (R.V.), rhythmically compressed with air from a compressor and a suitable valve mechanism. Water was pumped from this through a glass valve (P.V.) into an elastic system representing the arterial tree in which a variable resistance could be introduced by compressing a rubber segment enclosed in a glass tube (V.R.). The return flow then passed to an elastic bag (R.A.), representing the right atrium, the elastic properties of which could be altered by means of variable air pressure in a surrounding flask. This was connected to the "ventricle" via a glass valve representing the tricuspid

* In receipt of a Norwegian state grant.

$\dagger$ In receipt of a grant from the Medical Research Council. 


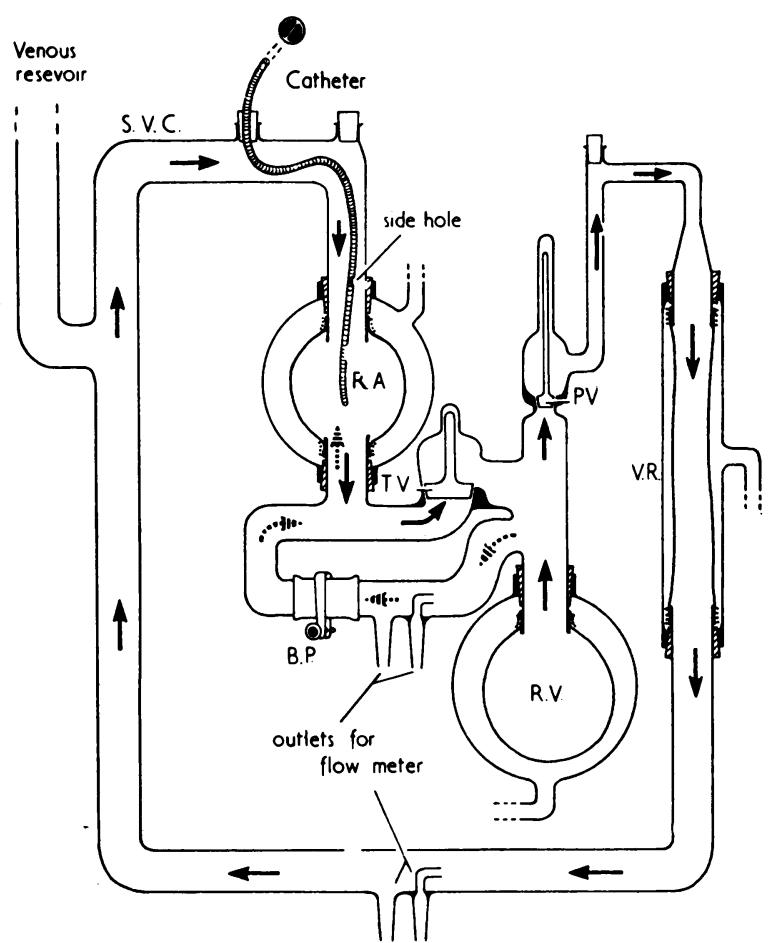

Fig. 1.-Diagram of the arrangement of the model heart used for testing the technique of measuring blood flow. R.A., " right atrium." T.V., " tricuspid valve." B.P., bye-pass of " tricuspid valve." R.V.," right ventricle.", P.V., " pulmonary valve." V.R., variable resistance.

S.V.C., "superior vena cava."

valve. Varying degrees of "tricuspid" leak could be introduced by means of a bye-pass tube and variable stop cock (B.P.). The amount of forward flow and regurgitation were determined by Pitot flow meters suitably placed in the circuit. The same double lumen catheter as was used in the patients was placed in the " atrium" so that the end hole was just inside the cavity and the side hole in the "superior vena cava."

\section{ExPerimental Results in the Heart Model}

The relationship between the differential pressures obtained between the two catheter lumina and the known regurgitant flow followed the formula for the Pitot type of flow meter, i.e. the flow plotted against the square root of the differential pressures was linear under conditions arranged to simulate as closely as possible those found in man (Fig. 2). The sensitivity was such that a back flow as low as $4 \mathrm{ml}$. a beat could be easily detected.

The position of the catheter in the " atrium" and "superior vena cava" did not greatly affect the resultant record provided that the tip did not lie too near the orifice of the "tricuspid valve." Withdrawing the catheter so that both the end holes were inside the "superior vena cava " gave a curve of very similar pattern to that before withdrawal and with only slight changes in magnitude (Fig. 3, A and B).

The effect of altering the venous pressure in the "atrium" is shown in Fig. 3, C and D: as the pressure was raised the regurgitant stream did not rise to such a high rate of flow at the beginning of ventricular systole but tended to persist longer so that the total volume of regurgitated blood 


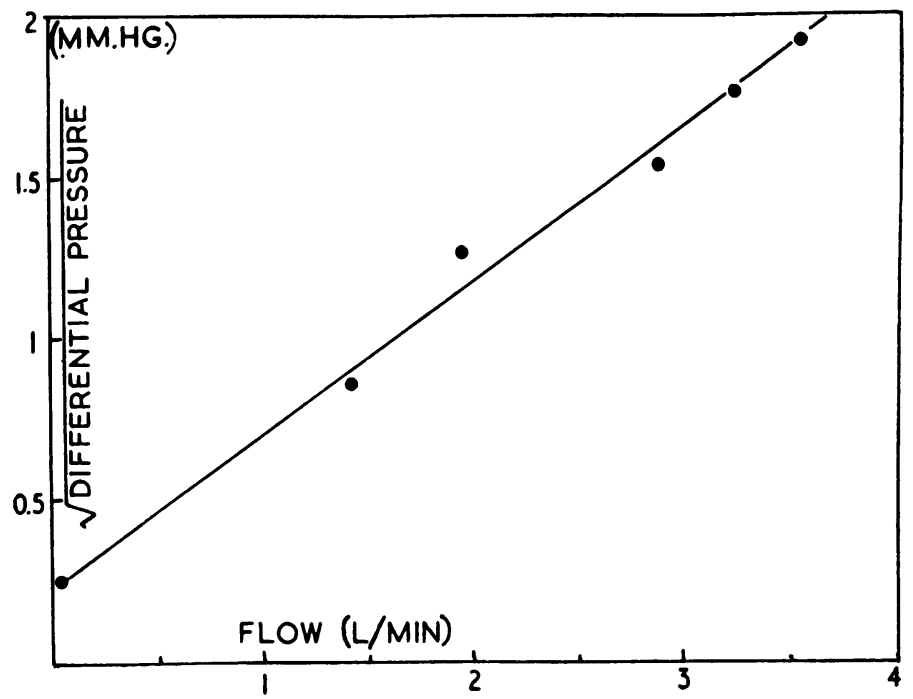

FIG. 2.-The relationship between the differential pressures recorded from the double lumen catheter and actual fluid flow as measured in the model heart.

was approximately the same. An increase in the rigidity of the system by placing a cork in the outlet of the venous reservoir to the air had a similar effect, which was more marked than the effect of increasing the venous pressure. The timing of the events in the cycle was recorded by a phonocardiographic tracing of the opening and closing of the "tricuspid" valve.

These results showed that the direction of the deflection of the curve always gave the direction of the actual flow and an indication of the amount of that flow throughout the cardiac cycle.

\section{Results In Patients}

The curves obtained from our patients in all cases closely resembled those produced in the model. The normal curve (Fig. 4A) showed a small reversed flow between the right atrium and superior

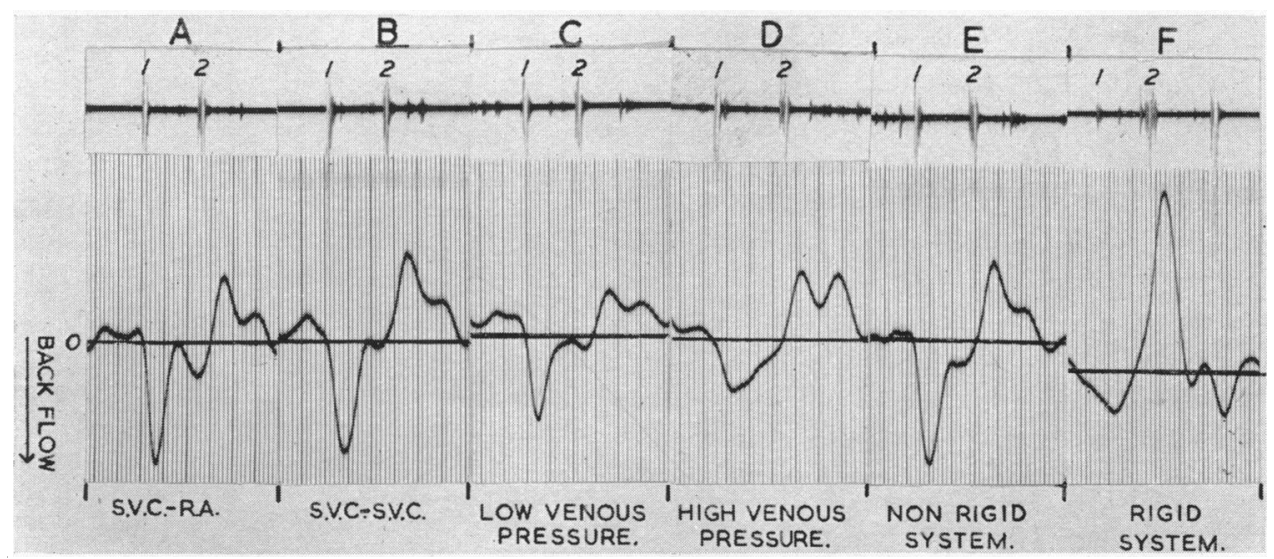

Fig. 3.-Flow patterns recorded by the double lumen catheter system in the model heart with a regurgitant stream equal to the forward output from the "right ventricle." (A) With the catheter tip in the "atrium" and side lumen in the "superior vena cava." (B) With the tip and side lumen in the "superior vena cava." (C) and (D) The effect of raising the venous pressure. (E) and (F) The effect of increasing the rigidity of the atrium and venous system. 


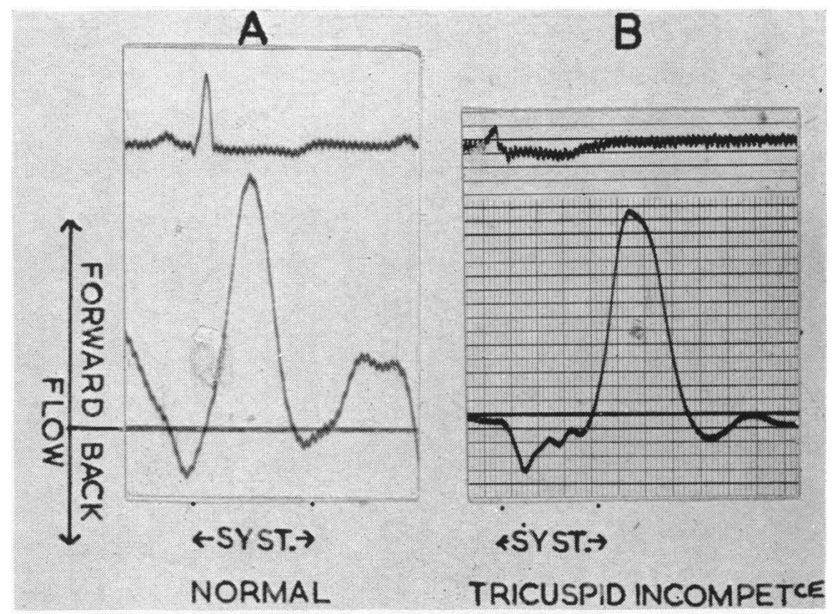

Fig. 4.- The blood flow between the superior vena cava and right atrium in a normal subject $(A)$ and one with tricuspid incompetence and high venous pressure (B).

vena cava during atrial systole. This rapidly gave way to a forward flow which reached its maximum in mid-ventricular systole and during descent of the base of the heart. At the end of systole the flow stopped and in some cases was slightly reversed during return of the base of the heart; the forward flow restarted early in diastole and continued until atrial systole. Most of the forward flow from the superior vena cava to the right atrium occurred during ventricular systole and descent of the base of the heart. A similar pattern was obtained between the inferior vena cava and right atrium.

In tricuspid insufficiency the normal pattern was greatly modified; as the tricuspid leak increased the forward flow during descent of the base decreased and in most cases was replaced by a reversed flow; where the venous pressure was not high and the venous system not tense this occurred for the most part early in systole (Fig. 5B), but in the presence of a higher venous pressure and a tense atrium the reversed flow tended to be more evenly distributed throughout systole (Fig. 4B). All the forward flow from the superior vena cava to the right atrium in these cases occurred during diastole.

The effect of exercise in increasing the amount of tricuspid leak is well shown in Fig. 5. The patient had chronic rheumatic heart disease with mitral stenosis and early cardiac failure. At rest there was a slight tricuspid leak with some impairment of forward flow in systole and a mean venous pressure of $10 \mathrm{~mm}$. $\mathrm{Hg}$. Two minutes exercise raised the mean venous pressure to $15 \mathrm{~mm}$. and produced a considerable increase in the regurgitant stream during ventricular systole.

\section{Discussion}

The experiments with the double lumen catheter in the model showed that the catheter-manometer system worked as a Pitot flow meter and gave a reliable indication of the presence of forward or reversed flow. The effect of changing the position of the catheter in the atrium and superior vena cava was surprisingly small; in man also the form of differential pressure curves did not vary greatly on withdrawal of the distal hole into the superior vena cava. A quantitative approach was possible in the model (Fig. 2) but owing to the presence of the inferior vena cava and other variable factors was not attempted in man.

The phasic variations in flow patterns in man closely resembled the curves obtained by Brecher (1954) in dogs by means of a bristle flow-meter. Normally the greater part of the forward flow of 


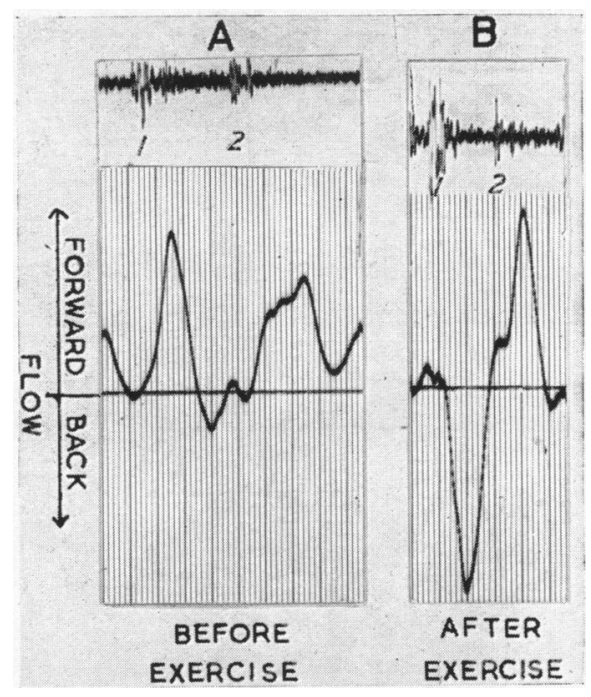

FIG. 5.-The effect of exercise on the degree of tricuspid incompetence in a patient with mitral stenosis and early cardiac failure.

blood from the great veins to the atrium occurs during ventricular systole and descent of the base of the heart; this forward flow correlates with the drop in atrial pressure ( $x$ descent) found in this part of the cardiac cycle. The blood flow stops, or even becomes momentarily reversed, at the time of the return of the base of the heart and starts again during diastole, but at a lower level than in systole. The effect of atrial contraction, although visible on the curves, is slight.

In tricuspid incompetence the normal phasic flow is greatly altered; where the leak is small the forward flow in systole is reduced by the regurgitant stream but the overall flow is still from the great veins to the atrium; a greater part of the forward flow then occurs in diastole. Factors that normally increase tricuspid incompetence in borderline cases may change one type of curve into the other. This was well shown in Fig. 5 where the patient on being exercised developed the typical flow pattern of gross tricuspid incompetence as well as a rise in venous pressure.

The effects of varying venous pressure and volume elasticity coefficient could easily be separated on the circulation model and the tracings obtained closely simulated curves made from patients in a variety of conditions. With a low venous pressure and a normal volume elasticity coefficient the backflow was greater and occurred earlier in systole than when the venous pressure was raised and the volume elasticity coefficient of the system increased, other factors remaining constant. A rising level of the venous pressure and diminished distensibility of the atrium tended to limit the amount of blood regurgitated in systole; the volume of backflow in turn affected the level of the mean venous pressure. An analysis of our curves in cases of severe tricuspid incompetence suggests that in many cases the backflow may equal or even exceed the forward cardiac output (Fig. 5B).

\section{SUMMARY}

A technique for recording the instantaneous changes in the blood flow from the superior vena cava to right atrium is described. In a model it permits of a good quantitative assessment of the rate of flow.

The blood flow in the superior vena cava and right atrium is recorded in patients with and without tricuspid incompetence. Normally the greater part of the forward flow from the superior vena cava 
to the right atrium takes place during ventricular systole and the descent of the base of the heart. In gross tricuspid incompetence the forward flow mainly occurs in diastole. With smaller degrees of tricuspid leak some forward flow may still occur at the end of systole.

The volume of regurgitated blood is closely related to the volume elasticity function of the right atrium and venous system.

In some patients with heart failure and functional tricuspid incompetence the amount of backflow may equal or exceed the forward cardiac output.

We should like to thank Professor McMichael for his continued help and advice and Dr. Korner for his valuable assistance.

\section{REFERENCES}

Brecher, G. A. (1954). Amer. J. Physiol., 176, 423.

Korner, P., and Shillingford, J. (1954). Brit. Heart J., 16, 447.

Müller, O., and Shillingford, J. (1954). Brit. Heart J., 16, 195.

- _ - (1955). J. Physiol., 126, in the press. 